REVISIONES

Rev Obstet Ginecol Venez. 2021; 81 (4): 365-376.

https://doi.org/10.51288/00810409

\title{
Sistema Internacional Yokohama para el informe de la citología mamaria. Academia Internacional de Citología
}

\author{
Morelva Toro de Méndez, ${ }^{1}$ (D) Ana Beatriz Azuaje de Inglessis. ${ }^{2}$
}

\begin{abstract}
RESUMEN
La citología por biopsia punción-aspiración con aguja fina forma parte de la triada de diagnóstico en la evaluación de la patología mamaria, junto con la clínica y la mamografía o ultrasonido. El sistema internacional Yokohama ha sido propuesto para informar los hallazgos de la citología mamaria por biopsia punción-aspiración con aguja fina, mediante 5 categorías de diagnóstico: inadecuada/insuficiente, benigna, atípica, sospechosa para malignidad y maligna, que incluyen definiciones, criterios citomorfológicos claves y sugerencias de manejo clínico. El objetivo de este artículo fue describir los lineamientos de este nuevo sistema de clasificación citológica mamaria para su implementación en esta región. La biopsia punción-aspiración con aguja fina es un método preciso y eficaz para diagnosticar las diferentes lesiones mamarias, lo cual requiere de una muestra celular adecuada y de una interpretación cito-morfológica precisa. La aplicación del sistema Yokohama estandarizado permitirá mejorar la evaluación, el diagnóstico y manejo clínico de la patología mamaria.
\end{abstract}

Palabras clave: Citología mamaria. Biopsia punción-aspiración con aguja fina. Sistema de informe Yokohama.

Yokohama International System for reporting breast cytology. The International Academy of Cytology

\section{SUMMARY}

Fine needle aspiration biopsy cytology is part of the diagnostic triad in the evaluation of breast pathology, along with clinical and mammography or ultrasound findings. The Yokohama international system of the International Agency for Cytology has been proposed to report fine needle puncture-aspiration biopsy breast cytology, through 5 diagnostic categories: Inadequatel insufficient, benign, atypical, suspicious for malignancy and malignant, including in each of these definitions, key cytological diagnostic criteria and suggestions for clinical management. The objective of this article was to know the guidelines of this new breast cytological classification system for its implementation in this region. Fine needle puncture-aspiration biopsy is a precise and effective method to diagnose different breast lesions, which requires an adequate cell sample and a precise cytomorphological interpretation. The application of standardized Yokohama system will allow to improve the evaluation, diagnosis and clinical management of the breast pathology.

Keywords: Breast cytology. Fine-needle aspiration biopsy (FNAB). Reporting system Yokohama.

\section{INTRODUCCIÓN}

La citología por biopsia punción-aspiración con aguja fina (BPAF) es un procedimiento de diagnóstico

${ }^{1}$ Bioanalista-citólogo. Doctora en Patología de los Tumores Humanos. Profesora Titular yCoordinadora del Grupo de Investigaciones Citológicas, Cátedra deCitología. Facultad de Farmacia y Bioanálisis. Universidad de Los Andes. Mérida. Venezuela. tmorelva@ ula.ve ${ }^{2}$ Médico Gineco-obstetra. Profesora Titular, Jubilada, del Departamento de Obstetricia y Ginecología de la Facultad de Medicina de la Universidad de Los Andes. Mérida. Venezuela. Correo de correspondencia: toromorelva@gmail.com

Forma de citar este artículo: Toro de Méndez M, Azuaje de Inglessis A B. Sistema Internacional Yokohama para el informe de la citología mamaria. Academia Internacional de Citología. Rev Obstet Ginecol Venez. 2021; 81(4):365-376. https://doi.org/10.51288/00810409 mínimamente invasivo y ampliamente utilizado en la evaluación inicial de la patología mamaria benigna y maligna, tanto de las lesiones que se presentan como masas palpables como de aquellas no palpables detectadas mediante imagenología, ya sea mamografía o ultrasonido, proporcionando información inmediata de utilidad clínica. Posee elevada efectividad, con sensibilidad de hasta el $96 \%$ y especificidad de $99 \%$; es bien aceptada por el paciente, femenino o masculino; no presenta contraindicaciones; es útil cuando se requiere de un diagnóstico rápido y forma parte de la triada de diagnóstico o triple test (prueba triple), junto con los hallazgos clínicos y de imagen. La BPAF 


\section{SISTEMA INTERNACIONAL YOKOHAMA PARA EL INFORME DE LA CITOLOGÍA MAMARIA. ACADEMIA INTERNACIONAL DE CITOLOGÍA}

mamaria es optimizada cuando se realiza guiada con ultrasonido y permite la obtención de material celular para pruebas auxiliares como marcadores pronóstico (1-4). También es utilizada cuando se requiere una evaluación on-site (Rapid On-site Evaluation, ROSE) en casos atípicos o sospechosos para malignidad, teniendo además gran utilidad en la reducción del porcentaje de muestras inadecuadas (5).

El Sistema Internacional Yokohama de la Agencia Internacional de Citología ha sido propuesto para informar los hallazgos en la citología mamaria por BPAF. Fue presentado por primera vez en 2016, durante el Congreso Internacional de Citología, en la ciudad de Yokohama, Japón, con la finalidad de desarrollar un sistema de informe citológico mamario estandarizado, de aceptación y aplicación universal, pues había sido diseñado incluyendo los algoritmos de manejo clínico para ser aplicable en todas las infraestructuras, para perfeccionar el informe citológico mamario estableciendo definiciones y criterios citomorfológicos característicos claros, permitiendo así una mejor comunicación entre citólogos y clínicos mediante la vinculación del informe citológico a las opciones de manejo clínico y, por último, para facilitar la investigación de la utilidad clínica de la citología en el estudio de las lesiones mamarias $(6,7)$.

Actualmente, ya está disponible el atlas de citología mamaria del Sistema Internacional Yokohama, estructurado en capítulos según las categorías de diagnóstico citológico, incluyendo en cada una de ellas definiciones, criterios citomorfológicos claves para diagnóstico con microfotografías explícitas, diagnóstico diferencial y ejemplos de informes según los hallazgos citológicos, así como un capítulo para sugerencias de manejo clínico y el riesgo de malignidad (Risk of Malignancy, ROM) para cada categoría; un capítulo para la citología de pezón y otro con las diferentes pruebas auxiliares para complementar el diagnóstico mediante el uso de la inmunocitoquímica y la biología molecular, siendo de fácil aplicación en bloques celulares elaborados a partir del mismo material obtenido por BPAF. Además, contiene un capítulo sobre la utilidad de la citología en base líquida mamaria, con la cual se elabora un frotis citológico para el estudio de la morfología. El material residual sirve para las pruebas auxiliares, cuando es requerido, sin necesidad de realizar otras tomas de muestra (8).

Entre los requerimientos cruciales de este sistema de clasificación para una correcta interpretación citológica y de verdadera utilidad clínica, se incluyen 1) un alto nivel en el desempeño y habilidad en la obtención de la muestra por BPAF, elaboración de los extendidos celulares y preservación apropiada del material celular obtenido, 2) un citopatólogo con conocimientos, bien entrenado y experimentado en la interpretación de los hallazgos citomorfológicos, especialmente cuando se trata de lesiones proliferativas mamarias que requieren de un diagnóstico diferencial y 3) una permanente correlación del triple test: citología, clínica e imagenología para determinar el manejo clínico apropiado para cada paciente (7). La BPAF puede ser usada para el diagnóstico de la mayoría de las lesiones mamarias palpables clínicamente y de las impalpables, pero detectadas por imagenología. Mientras que la biopsia con aguja gruesa, que es un procedimiento más invasivo, se prefiere cuando existen microcalcificaciones, que son frecuentemente asociadas a carcinoma in situ, cuando existen ciertas lesiones proliferativas o en el caso de lesiones difusas, discretas, no concluyentes, para excluir un carcinoma invasor y para realizar pruebas auxiliares. En este sentido, el ROM señalado para cada categoría de diagnóstico citológico debe ser considerado en el momento de la correlación del triple test (8).

En cuanto a la técnica de BPAF, se considera trascendental para una evaluación citológica exitosa, requiriendo habilidad y destreza para alcanzar con la aguja la lesión en estudio. En resumen, la lesión debe ser precisada, ya sea palpable o mediante ultrasonido, deberá fijarse con dos dedos, introducir la 
aguja calibre 25 y realizar varios pasajes dentro de la lesión aspirando durante el procedimiento, retirando hábilmente la aguja sin perder material, para proceder a elaborar correctamente al menos dos extendidos celulares, que deberán ser fijados: uno para tinción de Papanicolaou con alcohol y otro para Giemsa al aire, evitando la formación de artefactos. Todo este proceso debe realizarse en unos pocos minutos (8-13). Si se realiza una ROSE, esta permitirá una evaluación rápida para determinar la naturaleza de la lesión, si se requiere realizar una biopsia con aguja gruesa o de escisión para un diagnóstico definitivo o para pruebas auxiliares de inmunocitoquímica o moleculares. Aunque el principal aporte de ROSE es que puede evaluar la adecuación de la muestra y si no es satisfactoria, realizar nueva toma para continuar evaluando, hasta obtener un diagnóstico (5).

El informe estandarizado propuesto por el sistema Yokohama estaría estructurado así: un encabezado con la categoría de diagnóstico citológico, seguido de una descripción concisa de los hallazgos citológicos, señalando cuando sea posible, las características citomorfológicas claves de diagnóstico y por último, un diagnóstico tan específico de la lesión como sea posible. Si el diagnóstico es dudoso, se debe proporcionar los probables diagnósticos diferenciales. Cualquier discrepancia en el informe citológico debe ser resuelta en correlación con el triple test. Las categorías de diagnóstico citológico establecidas por el sistema Yokohama son: inadecuada/insuficiente, benigna, atípica, sospechosa de malignidad y maligna. En cada una de ellas se detalla la terminología, definiciones, criterios citomorfológicos claves, diagnóstico diferencial y sugerencias de manejo clínico, complementado con imágenes citológicas y ejemplificación de casos citológicos (8). El ROM para cada categoría, resumido de estudios recientes, es como sigue: para la categoría inadecuada/insuficiente oscila en el rango de 2,6 \% a 4,8\%; benigna $1,4 \%$ a $2,3 \%$; atípica de $13 \%$ a $15,7 \%$; sospechosa para malignidad $84,6 \%$ a $97,1 \%$ y para la categoría maligna entre $99,0 \%$ a $100 \%$, observándose claramente que va aumentando a medida que aumenta la severidad de las alteraciones citomorfológicas (3, 4), lo cual depende de las prácticas y la disponibilidad de los recursos médicos locales, por lo que el ROM actual deberá ser reestablecido por investigaciones futuras, en cada región. El objetivo de este artículo fue conocer los aspectos más resaltantes de este nuevo sistema de clasificación citológica mamaria, para su implementación en esta región. Para ello, se revisaron los artículos de publicación periódica relacionados con el origen del Sistema Yokohama $(6,7)$ y el atlas citológico, siendo este último la base del contenido expuesto, resumiendo del mismo los aspectos de mayor interés para los efectos del presente artículo (8).

\section{ASPECTOS RESALTANTES DEL SISTEMA}

La citología exfoliativa o por punción-aspiración con aguja fina es una metodología que se ha utilizado tradicional y ampliamente para la pesquisa de cáncer y lesiones precancerosas. Se caracteriza por ser sencilla, rápida y por poseer excelente rendimiento, ser costoefectiva y bien aceptada por el paciente (10). La estandarización del informe citológico en el estudio de las patologías de diversos órganos como el cuello uterino, tiroides, líquidos serosos, orina, entre otros, ha sido motivo de reuniones científicas en los últimos años, con el objeto de crear sistemas de clasificación citológica que conlleven al mejor manejo clínico del paciente y facilitar los estudios de investigación, así mismo, la calidad o adecuación de la muestra para el análisis citológico, en todos los casos, ha sido un aspecto de interés creciente, pues esta influye en la calidad de resultados y por ende, en la eficacia del examen citológico. La técnica y habilidad para la obtención de la muestra citológica, la preparación del extendido citológico y la preservación apropiada del material obtenido son consideradas aspectos cruciales para realizar un informe citológico certero (14 - 17). 


\section{SISTEMA INTERNACIONAL YOKOHAMA PARA EL INFORME DE LA CITOLOGÍA MAMARIA. ACADEMIA INTERNACIONAL DE CITOLOGÍA}

El Sistema Internacional Yokohama ha sido propuesto para el informe de los hallazgos citológicos de la BPAF de glándula mamaria. A continuación se expone un resumen de las pautas de este sistema de clasificación, establecidas en cada una de las 5 categorías de diagnóstico citológico para la patología mamaria, detalladas y ampliamente ejemplificadas en el atlas citológico, con microfotografías y propuestas de informe para cada categoría (8).

Categoría 1: Inadecuada/Insuficiente.

En esta categoría se incluyen los extendidos que no cumplen con los criterios de idoneidad. Una muestra adecuada es aquella que se considera suficiente para explicar la naturaleza de la lesión que se muestrea, el ejemplo más común es la presencia de células tumorales en la BPAF de una lesión nodular con sospecha de malignidad, clínica o por imagenología. Por tanto, en los casos con una masa sólida palpable o una lesión no palpable pero vista en la imagenología, es razonable requerir de entre 6 y 7 fragmentos de tejido epitelial, cada uno con al menos 10 - 20 células, para así poder evaluar la arquitectura de los grupos celulares y la presencia o ausencia de células mioepiteliales o núcleos bipolares, lo cual es considerado como una pauta de adecuación. Sin embargo, debe reconocerse que algunos tumores como el carcinoma lobulillar invasivo, pueden descamarse muy pocos fragmentos de tejidos o no descamar ninguno, presentándose solo algunas células atípicas dispersas, en cuyo caso la muestra se consideraría al menos "atípica". La presencia de un número mínimo de fragmentos de tejido celular epitelial es entonces un requerimiento para establecer la BPAF de una lesión nodular como adecuada, sin embargo, esto no garantiza un muestreo apropiado cuando se trata de lesiones no epiteliales como abscesos, quistes, lipomas o necrosis grasa. Por tanto, la valoración de la adecuación de la muestra por BPAF estará fundamentada en la calidad y cantidad de material celular presente.
Considerando lo anterior, esta categoría incluiría las muestras con escasa celularidad y las elaboradas o fijadas de forma defectuosa, lo que impediría una interpretación citomorfológica. También podrían ser inadecuados los extendidos citológicos muy finos o muy gruesos, obscurecidos por sangre y/o inflamación o aquellos con artefactos por aplastamiento del material obtenido y los secados al aire en muestras para coloración de Papanicolaou o por secado lento al aire para tinción de Giemsa. Los porcentajes elevados de muestras inadecuadas, de hasta 30,3\%, podrían ser un reflejo o la consecuencia por ejemplo de la no estandarización en la definición y criterios correspondientes a la adecuación de los especímenes celulares para estudio citológico, el uso de diferentes protocolos clínicos de obtención de la muestra celular incluyendo personal no entrenado apropiadamente, el muestreo de una mayor proporción de lesiones mamarias de bajo riesgo de malignidad, las poblaciones con diferentes características epidemiológicas (pesquisa en población general frente a población de riesgo con presencia de lesión palpable) y el uso o no de la evaluación ROSE, que reduce las muestras inadecuadas significativamente $(1,4,5,8)$.

Existen factores que afectan el porcentaje de muestras citológicas mamarias inadecuadas, siendo más frecuente en lesiones benignas que en las malignas. Entre ellos y particularmente los inherentes a la lesión, especialmente en las no palpables o en las de tamaño pequeño, aquellas poco proliferativas como las escirrosas o lobulillares, las microcalcificaciones sin masa palpable, necrosis, material infartado o supurativo que obscurece el material epitelial, todas ellas produciendo muestras hipocelulares o acelulares. También influyen los factores relacionados con la habilidad para la toma de muestra celular como lo es la inexperiencia, localización y fijación inapropiada de la lesión, uso de agujas inadecuadas, pasaje agresivo de la aguja sobre la lesión diana o no muestreo de esta, varios pinchazos y sobreaspiración, fallas en la 
presión negativa antes de retirar la aguja, incorrecta elaboración del o los extendidos celulares y/o fijación defectuosa con pérdida del material que se queda en la aguja o durante la elaboración, artefactos por aplastamiento del material y extendidos delgados o sanguinolentos, residuos del gel para el ultrasonido, coloración defectuosa, entre otros. Por todo ello, se prefiere el ROSE, que además de disminuir especialmente las muestras insuficientes, permite repetir la BPAF si no es satisfactoria y la clasificación de casos que requieren más bien de una biopsia con aguja gruesa $(4,5)$.

Entre las soluciones a todos estos inconvenientes se sugiere un entrenamiento apropiado y monitoreo de la toma de muestra por BPAF, un feedback continuo sobre las posibles causas de muestras inadecuadas a fin de solventarlas en las BPAF futuras y una correlación inmediata con la clínica e imagenología.

Para categorizar una muestra se recomienda el uso de los términos: inadecuado, en caso de que se deba a material mal extendido, preservado o fijado. Insuficiente, cuando es hipocelular o acelular en caso de una lesión palpable o cuando no se dispone de información clínica-imagenológica. Las características específicas de la muestra citológica que la hacen inadecuada o insuficiente siempre deben ser especificadas, a fin de que sean corregidas en las futuras prácticas.

Está claro que si se detecta alguna alteración morfológica como células epiteliales únicas, dispersas, con atipia nuclear significativa o necrosis en el fondo del frotis, entonces la muestra debe categorizarse al menos como "atípica". Por otra parte, existen varias situaciones clínicas en las que un extendido citológico puede ser adecuado, a pesar de la escasez de material epitelial y, además, porque los hallazgos citológicos son compatibles con los clínicos y de imagen, como es el caso de quistes, procesos inflamatorios (abscesos), lipoma, lesiones de células fusiformes (fibroblastos u otras células fusiformes o fragmentos de estroma), cicatriz radial (fragmentos de tejido esclerótico), necrosis grasa, fibroadenoma esclerótico o hialinizado. Esta categoría demuestra la necesidad imperiosa de obtener una muestra celular de elevada calidad, a fin de lograr un informe citológico certero, que permita contribuir en el esclarecimiento de la patología mamaria, basado en el aporte oportuno de datos clínicos y de imagenología. El algoritmo de manejo clínico sugerido para esta categoría consiste primero en realizar la correlación del triple test; si existieran discrepancias en este, se debe sugerir repetir la BPAF o realizar una biopsia con aguja gruesa, si existe alguna duda en el entorno clínico multidisciplinario. Si se trata de un problema técnico, repetir la BPAF, atendiendo el error para no repetirlo. Si existe una imagen indeterminada o atípica por mamografía o por ultrasonido, realizar biopsia de escisión. En segundo lugar, si la probabilidad de malignidad es baja se sugiere evaluar con el triple test de 3 a 6 meses. Es posible que el ROSE aclare cualquier duda o incongruencia (18).

Categoría 2: Benigna.

Un diagnóstico por BPAF de citología mamaria benigna se realiza en casos que muestran características citomorfológicas inequívocas de benignidad, que pueden o no ser diagnóstico de una lesión benigna específica. La citología mamaria benigna incluye el tejido mamario normal, los procesos inflamatorios (mastitis aguda, granulomatosa y abscesos), quistes, cambios fibroquísticos y fibrosis, las lesiones proliferativas benignas como la hiperplasia epitelial simple, fibroadenoma, papiloma intraductal y tumor phylodes benigno. También, los cambios por lactancia, ginecomastia, posradiación, cicatriz radial y lesiones esclerosantes complejas, adenosis y adenosis esclerosante, necrosis grasa y nódulo linfático intramamario. 


\section{SISTEMA INTERNACIONAL YOKOHAMA PARA EL INFORME DE LA CITOLOGÍA MAMARIA. ACADEMIA INTERNACIONAL DE CITOLOGÍA}

Los criterios citomorfológicos generales incluyen, tanto para el tejido mamario normal como para las diferentes lesiones mamarias benignas, la presencia de células epiteliales agrupadas en fragmentos epiteliales en monocapa, de tamaño variable, bien cohesivos y con mínima atipia nuclear, preservando la relación núcleo/ citoplasma y un nucléolo pequeño regular. El fondo generalmente es limpio, proteináceo o inflamatorio, con células mioepiteliales entremezcladas con las epiteliales o como núcleos bipolares, pueden verse células de metaplasia apocrina en placas, células inflamatorias y/o macrófagos, que, dependiendo de la patología existente pueden verse combinados, conformando un cuadro citológico característico. La presencia de células aisladas y dispersas no es frecuente. En las lesiones mamarias proliferativas benignas se requiere de una evaluación citológica cuidadosa, especialmente si existen atipias discretas, siempre en correlación con la clínica e imagenología, a fin de evitar falsos positivos.

Eldiagnóstico diferencial se presenta básicamente entre los casos de lesiones con proliferación epitelial y/o estromal, principalmente la hiperplasia ductal atípica, el carcinoma ductal in situ de bajo grado y variantes como sólido, papilar o micropapilar, los carcinomas de tipos histopatológicos específicos considerando el material epitelial y/o estromal predominante, el carcinoma de alto grado no específico y la neoplasia lobular.

Las lesiones benignas en el triple test pueden vigilarse con exploración física e imagenología para asegurar que no ha ocurrido ningún cambio, en un intervalo de 3 a 6 meses. Si dicha lesión permanece estable, la paciente puede regresar a la pesquisa de rutina. Ante cualquier cambio durante el seguimiento clínico o en imágenes, se realiza una nueva BPAF y si esta resulta discordante con los hallazgos clínicos o imagenológicos, se debe realizar biopsia con aguja gruesa o considerar la biopsia de escisión simple (18).
Categoría 3: Atípica.

Esta categoría incluye la presencia de características citomorfológicas vistas predominantemente en procesos o lesiones benignas, pero con la adición de algunas otras características que son poco frecuentes en dichas lesiones y que además pueden verse también en lesiones malignas. Por tanto, es una interpretación incierta que requiere de mayor investigación (13), aunque se considera que esta categoría es sugestiva de carcinoma ductal in situ de bajo grado.

En la citología mamaria atípica se observan las siguientes alteraciones citomorfológicas generales: hipercelularidad, representada por grandes placas de tejido epitelial con patrón que puede ser irregular, cribiforme, papilar o micropapliar, y/o estromal, con amontonamiento celular incrementado y discreto grado de atipia nuclear como agrandamiento o pleomorfismo, asociado a la aparición de células atípicas bien preservadas, aisladas y dispersas, con agrandamiento o pleomorfismo nuclear. Es notoria la existencia de pocas células mioepiteliales y núcleos bipolares. El fondo puede ser variable, aunque la presencia de necrosis está asociada a carcinoma ductal in situ de bajo grado. De ser posible, se debe sugerir una interpretación diagnóstica específica y si no, indicarse las posibles lesiones incluidas dentro del diagnóstico diferencial, considerando la información clínica y de imagen aportada.

Esta categoría atípica está influenciada por factores variables como: 1) de origen técnico, relacionados con la habilidad y destreza en la obtención de material celular, elaboración de extendidos y proceso de fijación. Este tipo de limitaciones técnicas juegan un papel importante en el porcentaje de frecuencia de citologías mamarias atípicas, ya que pueden ser la causa de muestras con hipocelularidad, material celular suficiente pero incorrectamente extendido o fijado, que limita su análisis e interpretación, 
especialmente las alteraciones nucleares, lo que podría conducir a diagnósticos erróneos. 2) Los factores relacionados con la interpretación morfológica atípica, que dependen del entrenamiento y la experiencia del citólogo, lo cual es crucial para realizar una certera interpretación citológica, al orientar los hallazgos hacia una lesión benigna florida o por el contrario, a una neoplasia. Un citólogo experimentado es capaz de reconocer patrones citomorfológicos característicos de las diversas lesiones mamarias benignas, tales como el fibroadenoma o el papiloma intraductal y evaluar la posible existencia de atipia celular, para determinar si están dentro del grado aceptable para cada lesión específica o no, es decir, determinar la posibilidad de existencia de una lesión neoplásica. 3) Las limitaciones inherentes a la propia lesión podrían producir problemas de interpretación aún para los citólogos experimentados, debido básicamente, a la superposición de alteraciones morfológicas propias y vistas tanto en lesiones proliferativas benignas como en las neoplásicas. Por lo que es necesario tener claras las características citomorfológicas entre las lesiones mamarias benignas proliferativas, para detectar la existencia de posibles atipias mamarias asociadas a una lesión maligna, evitando así, un aumento del porcentaje de citologías mamarias de significado clínico incierto.

Entre las lesiones mamarias específicas que pueden causar dificultades en su correcta interpretación citológica y que podrían conllevar a un resultado atípico se encuentran el fibroadenoma mamario, papiloma intraductal, la hiperplasia epitelial usual (típica), la adenosis esclerosante, lesión fibroepitelial con abundante celularidad estromal, células apocrinas atípicas, la neoplasia lobular, el carcinoma ductal in situ de bajo grado, carcinoma invasor de bajo grado, tumor phylodes boderline o de bajo grado, adenomioepitelioma, miofibroblastoma, fibromatosis y la fascitis nodular. La presencia de núcleos bipolares y de células mioepiteliales orienta hacia una lesión benigna, mientras que su desaparición progresiva, combinada con atipias en células aisladas son más bien a favor de lesiones neoplásicas in situ, lobulares o invasoras. Por tanto, el diagnóstico diferencial es fundamental para evitar un aumento de las tasas de informes citológicos mamarios atípicos. La información clínica y de imagenología apropiada contribuye claramente en una correcta interpretación de los hallazgos citológicos atípicos.

El manejo clínico de una paciente con citología mamaria atípica estaría basado primero en la correlación del triple test y si los hallazgos coinciden con ser más hacia la benignidad se debe repetir la BPAF y si esta resulta benigna, corresponde seguimiento a los 3 - 6 meses. La biopsia con aguja gruesa guiada estaría indicada cuando los hallazgos clínicos/imagen sean indeterminados o sospechosos. Mientras que la biopsia escisional sería apropiada cuando existe una lesión proliferativa con atipias en el resultado de la biopsia con aguja gruesa (18).

Categoría 4: Sospechosa de malignidad.

El término sospechoso de malignidad en la citología mamaria está asociado a la presencia de algunas características citomorfológicas usualmente observadas en la neoplasia maligna, pero que son insuficientes, en cantidad y calidad, para categorizarla definitivamente como maligna y sería sugestiva de carcinoma invasor. El tipo histopatológico de malignidad que se sospecha debe ser sugerido, si es posible. Esta categoría está sujeta a una amplia variabilidad de carácter técnico, conceptual y de criterios citomorfológicos, de manejo clínico así como interobservador, al igual que la categoría atípica. A pesar de ello, su valor predictivo positivo para malignidad es elevado.

Los criterios citomorfológicos generales para esta categoría básicamente son la hipercelularidad, bien representada por un patrón de células pequeñas aisladas, intactas y bien preservadas, dispersas o dispuestas "en fila india", con discretas alteraciones nucleares 


\section{SISTEMA INTERNACIONAL YOKOHAMA PARA EL INFORME DE LA CITOLOGÍA MAMARIA. ACADEMIA INTERNACIONAL DE CITOLOGÍA}

(aumento del tamaño con incremento de la relación núcleo/citoplasma, pleomorfismo e hipercromasia) y citoplasma excéntrico, plasmocitoide, o también puede observarse un patrón consistente con grandes fragmentos de tejido epitelial tridimensionales sólido, cribiforme, papilar o micropapilar o una mezcla de ambos patrones. También, pequeños fragmentos de tejido con pérdida de la cohesividad, poca/intermedia atipia nuclear, nucléolos prominentes y calcificaciones rodeadas de células epiteliales atípicas, entremezcladas con necrosis granular, proteináceo o restos necróticos, sugiriendo la posible existencia de un carcinoma ductal in situ de alto grado. La presencia de células mioepiteliales y de núcleos bipolares es cada vez menor.

Las lesiones mamarias específicas que podrían conllevar a un informe citológico sospechoso son el carcinoma ductal in situ, de bajo y alto grado, aunque es controversial, y un cáncer invasor no puede ser excluido con certeza. Además, cualquier lesión proliferativa como la hiperplasia ductal atípica, ya que existe superposición de criterios citomorfológicos con el carcinoma in situ. El diagnóstico diferencial incluye la hiperplasia epitelial usual, fragmentos de tejido epitelial tridimensional complejo y el carcinoma invasor de bajo grado. La elaboración y fijación del extendido celular defectuosas podrían conllevar también a una categorización sospechosa, ya que los fragmentos de tejido podrían desintegrase durante la elaboración del frotis o formarse artefactos por fijación defectuosa. El objetivo es reconocer las características citomorfológicas de un posible carcinoma y evitar un resultado falso negativo de cáncer en asociación con lesiones proliferativas o resultados falsos positivos en lesiones proliferativas atípicas, que conducirían a cirugías agresivas.

En principio, la sugerencia de manejo clínico más prudente, para evitar cirugías innecesarias, es la biopsia con aguja gruesa. Dependiendo del criterio clínico y hallazgos mamográficos, es válida la biopsia de escisión. También es posible la resonancia magnética en lesiones no palpables (18).

Categoría 5: Maligna.

Esta categoría es una afirmación inequívoca de que el material celular posee características citomorfológicas compatibles con malignidad y debe indicarse el tipo histológico identificado siempre que sea posible. Esta interpretación tiene elevado valor predictivo positivo cuando se aplican los criterios citomorfológicos específicos claves. Los resultados falsos positivos son muy raros y serían por una mala interpretación de lesiones proliferativas floridas; los falsos negativos corresponderían a muestras con escaso material maligno, entremezclado con células benignas. La interpretación citológica de malignidad solo debe realizarse cuando se identifican criterios citomorfológicos de diagnóstico irrefutables, sin discrepancias con posibles lesiones benignas.

Las alteraciones citomorfológicas compatibles con neoplasia maligna incluyen hipercelularidad con un patrón celular epitelial y/o estromal dispuesto en pequeños fragmentos de tejido, con amontonamiento celular, pérdida de la cohesividad y marcada superposición nuclear, o un patrón de células intactas, aisladas, dispersas con prominente megalocariosis, pleomorfismo celular y nuclear, cromatina irregular o hipercromasia y macronucléolos. El fondo de frotis es variable y está relacionado con el tipo específico de tumor. Los diferentes tipos histopatológicos de cáncer de mama presentan características clínicas, histopatológicas y cuadros cito-morfológicos específicos actualmente bien conocidos.

Entre las neoplasias mamarias específicas para un informe citológico de malignidad se encuentran el carcinoma invasor de tipo no específico, el cual puede ser de bajo o de alto grado y representa el $75 \%$ de los casos. También el carcinoma ductal in situ de alto grado, el carcinoma lobular invasor, 
carcinoma con características de medular, carcinoma mucinoso, carcinoma micropapilar invasor, carcinoma metaplásico, carcinoma secretor, carcinoma de células claras rico en glucógeno, carcinoma adeno-quístico, carcinoma con diferenciación apocrina, carcinoma con características de neuroendocrino, linfoma no Hodgkin, angiosarcomas y carcinomas metastásicos.

Una interpretación citológica de malignidad solo debe realizarse cuando existe una combinación de criterios citomorfológicos de diagnóstico certero, considerando el patrón de tejido epitelial prevalente y el grado de atipia nuclear, por lo que el diagnóstico diferencial se basa principalmente en la diferenciación de posibles lesiones benignas, que pudieran mostrar algunos criterios de malignidad en comparación con la contraparte maligna, marcada por los criterios citomorfológicos neoplásicos, tanto generales como específicos para los diferentes tipos histopatológicos de tumor, como el carcinoma ductal in situ de alto grado, carcinoma invasor de alto grado, carcinoma de tipo no especial de alto grado, carcinoma lobular clásico, lesiones benignas proliferativas con grados variables de atipia nuclear como en fibroadenoma, papiloma intraductal, cicatriz radial o cambios citoplasmáticos apocrinos, tumor de células granulares, linfomas, melanoma y otros tumores primarios específicos o metastásicos. Evidentemente, tanto la historia, impresión clínica, hallazgos de mamografía o ultrasonido y una muestra celular adecuada son imprescindibles para una segura interpretación citológica de malignidad.

La biopsia con aguja gruesa o quirúrgica son el primer paso, incluyendo el estudio de nódulos linfáticos axilares. Si existe correlación clínico/imagenológica, se recomienda solicitar pruebas auxiliares que apoyen el diagnóstico o para una mejor clasificación tumoral y de pronóstico (18).

Citología del pezón

Una secreción por el pezón unilateral, espontánea, persistente y que no esté relacionada con embarazo o lactancia es un hallazgo anormal, pudiendo estar asociada a un desorden hormonal, ectasia ductal, papiloma intraductal o cáncer (19). La secreción se toma en una lámina portaobjeto, sin tocar la piel, se recomienda elaborar al menos dos extendidos y fijar uno con alcohol para tinción de Papanicolalou y el otro al aire para Giemsa, de igual forma que se realiza para las muestras celulares obtenidas por BPAF. Una secreción por el pezón puede estar relacionada con los cambios por lactancia, con un proceso infeccioso/ inflamatorio, un papiloma intraductal $\mathrm{y}$, aunque una interpretación citológica de malignidad es rara, podría tratarse de un carcinoma, ya sea de tipo ductal in situ de bajo grado, con mucha dificultad de precisar, o invasor.

Los hallazgos citomorfológicos en secreciones por el pezón de lesiones benignas consisten en histiocitos, células en diferentes grados degenerativos, fragmentos de tejido epitelial papilariforme y siderófagos, sobre fondo proteináceo, lipídico, inflamatorio, sanguinolento, dependiendo de la patología asociada. En el caso de malignidad son similares a las observadas en muestras por BPAF, que, en secreciones por el pezón se recomienda clasificar como sospechosa de malignidad.

El manejo de las lesiones mamarias con secreción por el pezón requiere de un balance para evitar procedimientos innecesarios. Si la secreción está asociada con anormalidades clínicas o en la imagenología, esta requiere de mayor investigación con BPAF o biopsia con aguja gruesa. Los casos con fragmentos de tejido tipo papilar, pequeños, redondeados, con atipia leve presentan cierto grado de dificultad, ya que la atipia puede representar un cambio degenerativo. En esta situación, si no hay hallazgos clínicos o de imagen sospechosos, la conducta sugerida es el seguimiento. Si la secreción por el pezón continúa o se vuelve sanguinolenta, la escisión del conducto debe ser 


\section{SISTEMA INTERNACIONAL YOKOHAMA PARA EL INFORME DE LA CITOLOGÍA MAMARIA. ACADEMIA INTERNACIONAL DE CITOLOGÍA}

considerada. Un resultado citológico negativo no excluye el cáncer intraductal o invasor, especialmente por encima de los 50 años de edad.

Por otra parte, la enfermedad de Paget puede presentarse con secreción por el pezón, una apariencia eritematosa, ulceración o con la destrucción del pezón. Casi siempre hay carcinoma ductal in situ subyacente, generalmente de alto grado nuclear. El carcinoma invasor asociado se identifica en aproximadamente la mitad de los pacientes y puede ser palpable o mostrar en las imágenes calcificaciones o una masa.

Las características citomorfológicas corresponden a fragmentos tisulares diminutos, poco cohesivos con núcleos hipercrómicos y células de Paget individuales, redondas u ovaladas, con núcleo pleomórfico de gran tamaño, excéntrico, cromatina gruesa, nucléolo prominente y abundante citoplasma claro, de variable densidad. Pueden estar presentes células inflamatorias, residuos necróticos y restos queratóticos entre las células escamosas. En algunos casos solo hay escasas células atípicas y la atipia reactiva escamosa puede llevar a un diagnóstico incorrecto de carcinoma escamoso. En estos casos, la información clínica de cualquier hallazgo en el pezón es de gran ayuda. La evaluación de una lesión subareolar puede ser con BPAF guiada con ultrasonido. El melanoma es el principal motivo para diagnóstico diferencial, aunque raro en el pezón. El estudio inmunocitoquímico en bloques celulares contribuye con el diagnóstico definitivo y diferencial con melanoma y enfermedad de Bowen. La biopsia confirma el tipo de lesión (18).

Técnicas auxiliares en la evaluación y manejo de la patología mamaría

La citología mamaria mediante BPAF se apoya en pruebas especiales como la inmunocitoquímica y las técnicas moleculares como la hibridización in situ con fluorescencia (Fluorescent In Situ Hybridization, FISH) para el diagnóstico y clasificación de los cánceres de mama y para el pronóstico y predicción de la respuesta al tratamiento. Estas metodologías son sugeridas por el Sistema Internacional Yokohama ya que son de gran utilidad para aclarar el diagnóstico en casos desafiantes, atípicos y sospechosos para malignidad, en la resolución de casos con diagnóstico diferencial entre lesiones benignas y malignas y para establecer el subtipo histológico de cáncer, especialmente en el reto particular de las lesiones de células fusiformes, así como la metástasis de otras neoplasias en la mama. Las pruebas siempre deben interpretarse dentro de un contexto clínico-citomorfológico (20).

La citología en base líquida también es utilizada para el estudio de la patología mamaria. Requiere de un entrenamiento previo y aunque los cambios citomorfológicos son menores, posee una sensibilidad y especificidad similares a las preparaciones convencionales (21).

\section{CONCLUSIONES}

Actualmente, la citología por biopsia punciónaspiración con aguja fina (BPAF) se utiliza como primera línea de evaluación e investigación de la patología benigna y maligna de la glándula mamaria tanto femenina como masculina, en muchos países desarrollados y en algunos países en desarrollo. Forma parte de la triada de diagnóstico, junto con la clínica y la mamografía/ultrasonido. La BPAF es un método preciso y eficaz para diagnosticar las lesiones mamarias de diferente naturaleza, para lo cual requiere de una muestra celular adecuada para una interpretación citomorfológica precisa. El Sistema Internacional Yokohama de la Sociedad Internacional de Citología, es apropiado para informar con mayor precisión los hallazgos citológicos en la valoración clínica de las diferentes lesiones mamarias. La aplicación del Sistema Internacional Yokohama estandarizado permitirá mejorar la evaluación, el diagnóstico y manejo clínico de la patología mamaria. 


\section{AGRADECIMIENTO}

Las autoras expresan su agradecimiento al Profesor Dr. Fernando Schmitt, del Departamento de Patología y Oncología de la Facultad de Medicina de la Universidad de Oporto, Portugal y Presidente de la Sociedad Internacional de Patología Mamaria, por su gentileza al proporcionarnos la bibliografía requerida.

\section{REFERENCIAS}

1. Hoda RS, Brachtel EF. International Academy of Cytology Yokohama System for Reporting Breast FineNeedle Aspiration Biopsy Cytopathology: A Review of Predictive Values and Risks of Malignancy. Acta Cytol. 2019; 63(4):292-301. doi: 10.1159/000500704.

2. Hoda RS, Arpin Iii RN, Gottumukkala RV, Hughes KS, Ly A, Brachtel EF. Diagnostic Value of Fine-Needle Aspiration in Male Breast Lesions. Acta Cytol. 2019; 63(4):319-327. doi: 10.1159/000494486.

3. Montezuma D, Malheiros D, Schmitt FC. Breast Fine Needle Aspiration Biopsy Cytology Using the Newly Proposed IAC Yokohama System for Reporting Breast Cytopathology: The Experience of a Single Institution. Acta Cytol. 2019:1-6. doi: 10.1159/000492638.

4. Wong S, Rickard M, Earls P, Arnold L, Bako B, Field AS. The International Academy of Cytology Yokohama System for Reporting Breast Fine Needle Aspiration Biopsy Cytopathology: A Single Institutional Retrospective Study of the Application of the System Categories and the Impact of Rapid Onsite Evaluation. Acta Cytol. 2019; 63(4):280-291. doi: 10.1159/000500191.

5. Cai G, Adeniran AJ, editores. Rapid On-site Evaluation (ROSE). A Practical Guide. 1th ed. Nueva York: Springer; 2019.

6. FieldAS, SchmittF, Vielh P. IAC Standardized Reporting of Breast Fine-Needle Aspiration Biopsy Cytology. Acta Cytol. 2017; 61(1):3-6. doi: 10.1159/000450880.

7. Field AS, Raymond WA, Rickard M, Arnold L, Brachtel EF, Chaiwun B, et al. The International Academy of Cytology Yokohama System for Reporting Breast FineNeedle Aspiration Biopsy Cytopathology. Acta Cytol. 2019; 63(4):257-273. doi: 10.1159/000499509.

8. Field AS, Raymond WA, Schmitt F, editores. The
International Academy of Cytology Yokohama System for Reporting Breast Fine Needle Aspiration Biopsy Cytopathology.1th ed. Nueva York: Springer Nature. 2020.

9. Kocjan G, Gray W, Levine T, Kardum-Skelin I, Vielh P. Diagnostic Cytopathology Essentials. 1th ed. Amsterdan: Elsevier. 2013.

10. Ly A. Fine Needle Aspiration Biopsy Technique and Specimen Handling. En: Cibas ES y Ducatman BS, editores. Cytology. Diagnostic principles and clinical correlates. 5th ed. Amsterdam: Elsevier; 2021. p. 238-53.

11. Field A, Zarka M, editores. Practical cytopathology: a diagnostic approach to fine needle aspiration biopsy. 1th ed. Amsterdam: Elsevier; 2016.

12. Orell SR, Philippe V. En: Fine Needle Aspiration Cytology. The techniques of FNA cytology. 5th ed. Amsterdam: Elsevier: 2011. p. 20 - 9.

13. Orell SR, Sterrell .GF. Cap 7: Breast. En: Fine Needle Aspiration Cytology. 5th ed. Amsterdam: Elsevier. 2012. p.168 - 221.

14. Nayar R, Wilbur DC, editores. The Bethesda System for Reporting Cervical Cytology Definitions, Criteria, and Explanatory Notes. 3th ed. Nueva York: Springer Nature. 2015.

15. Ali SZ, Cibas ES, editores. The Bethesda System for Reporting Thyroid Cytopathology. Definitions, Criteria, and Explanatory Notes. 2th ed. Nueva York: Springer Nature. 2018.

16. Chandra A, Crothers B, Kurtycz D, Schmitt F, editores. The International System for Serous Fluid Cytopathology. 1th ed. Nueva York: Springer Nature. 2020.

17. Barkan GA,Wojcik EM, Nayar R, Savic-Prince S, Quek M.L, Kurtycz DFI, et al. The Paris System for Reporting Urinary Cytology. Acta Cytologica. 2016; 60:185-97. doi.org/10.1159/000446270

18. Cohen-Hallaleh R, Rickard MT, Lim E, Raymond WA, Segara D, Arnold L, et al. Clinical management. En: Andrew S. Field AS, Raymond WA, Schmitt F, editores. The International Academy of Cytology Yokohama System for Reporting Breast Fine Needle Aspiration Biopsy Cytopathology. Nueva York: Springer Nature. 2020. p.175-180.

19. Lee AHS, Field AS. Nipple Cytopathology. En: Andrew S. Field AS, Raymond WA, Schmitt F, editores. The International Academy of Cytology Yokohama System 
for Reporting Breast Fine Needle Aspiration Biopsy Cytopathology. Nueva York: Springer Nature. 2020. p. $131-80$.

20. Beca F, Schmitt F. Role of Ancillary Tests in Breast Fine Needle Aspiration Biopsy Cytopathology. En: Field AS, Raymond WA, Schmitt F, editores. The International Academy of Cytology Yokohama System for Reporting Breast Fine Needle Aspiration Biopsy Cytopathology. Nueva York: Springer Nature. 2020. p. $137-57$.
21. Schmitt F, Hoda RS. Fine Needle Aspiration Biopsy Cytopathology of the Breast Utilizing Liquid-Based Preparations. En: Field AS, Raymond WA, Schmitt F, editores. The International Academy of Cytology Yokohama System for Reporting Breast Fine Needle Aspiration Biopsy Cytopathology. Nueva York: Springer Nature. 2020. p. 159 - 74.

Recibido 6 de abril de 2021

Aprobado 3 de julio de 2021 